\title{
Compensation of time-varying clock-offset in a LBL navigation
}

\author{
Yohannes S.M. Simamora ${ }^{1}$, Harijono A. Tjokronegoro ${ }^{2}$, Edi Leksono ${ }^{3}$, Irsan S. Brodjonegoro ${ }^{4}$ \\ ${ }^{1,2,3}$ Engineering Physics Research Group, Institut Teknologi Bandung, Indonesia \\ ${ }^{4}$ Offshore Engineering Research Group, Institut Teknologi Bandung, Indonesia \\ ${ }^{1}$ Department of Mechanical Engineering, Politeknik Purbaya, Indonesia
}

\begin{tabular}{|c|c|}
\hline Article Info & ABSTRACT \\
\hline Article history: & \multirow{8}{*}{$\begin{array}{l}\text { This paper presents compensation of the clock-offset in a long baseline (LBL) } \\
\text { navigation. It departs from the existing literature mainly in dealing with a time-varying } \\
\text { clock-offset, i.e. the clock-rate drifts over the time. Specifically, the clock-offset } \\
\text { dynamics are introduced to the ToFs as an autoregressive filter. Subsequently, } \\
\text { interactions among the now biased ToFs and the kinematics of an autonomous } \\
\text { underwater vehicle (AUV)-the navigation subject-are represented in a state-space } \\
\text { form. Implementing the so-called graphic approach, minimum sensor requirement } \\
\text { for this system's observability is then explicated. Finally, a standard discrete Kalman } \\
\text { filter is deployed as the state estimator. By simulation, it is demonstrated that the } \\
\text { estimator manages to compensate the offset and to provide localization with less than } \\
1 \mathrm{~m} \text { accuracy. }\end{array}$} \\
\hline Received Aug 7, 2019 & \\
\hline Revised Oct 3, 2019 & \\
\hline Accepted Dec 11, 2019 & \\
\hline Keywords: & \\
\hline Clock-offset & \\
\hline Long baseline & \\
\hline Sonar & \\
\hline
\end{tabular}

State estimation

Time-of-flight

\section{Corresponding Author:}

Yohannes S.M. Simamora,

Engineering Physics Research Group,

Institut Teknologi Bandung,

Jl. Ganesha 10 Bandung 40132 Indonesia.

Email: simamora@alumni.itb.ac.id

\section{INTRODUCTION}

In navigation and localization [1,2], a long baseline (LBL) acoustic positioning system serves as position reference in the water. This is much similar to the role of the global positioning systems (GPS) in the terrestrial and aerial applications. In this sense, position of a navigation subject is computed based on a set of ranges between each acoustic transponder and the subject. Each range is obtained by measuring traveling time of an acoustic wave from the transponder in question to the subject, either in a two-way travel time (TWTT) or one-way travel-time (OWTT) configuration [3]. The acquired time multiplied by propagation speed of the wave. This method is commonly termed as time-of-flight (ToF) [4] measurement.

As implied by the above description, there are several prerequisites for an ideal ToF. First, the wave in question must travel in a line-of-sight (LoS). Second, the measurement points are at stationary positions during the ToF. Third, the clocks used in recording send and receive times must be in agreement with a shared reference, e.g. the absolute clock. For an individual clock, this synchronization issue is related to time, frequency, and phase [5].

If one of the aforementioned conditions does not hold, the ToF will result in a pseudorange, i.e. a biased range. A ToF with non-LoS condition may occur both in electromagnetic [6] and underwater acoustic $[7,8]$ waves. On the other hand, the case of non-stationary measurement point may arise only in 
acoustic based ToFs. This is due to the wave's slow propagation speed [9]. Some scenarios related to this problem have been addressed in the literature, e.g. queuing ToFs [10-12] and moving navigation subject with non-negligible speed $[13,14]$.

Meanwhile, a divergence to the third condition would introduce clock-offset to the ToF. Since wave speed $(\approx 1500 \mathrm{~m} / \mathrm{s})$ is the multiplying factor in a ToF, the presence of even a minuscule offset would propagate a noticeable bias to the pseudorange. In one recent literature [15], this problem has been carried out in an elegant state-space formulation with some promising results. This problem is also brought up in [13] along with the non-stationary ToF measurement. In both aforementioned works, the clock-offset is considered as a constant. Nonetheless, for a common clock with quartz oscillator, its decreasing performance is partly due to aging, temperature, pressure, and humidity $[16,17]$. In this sense, an existing clock-offset will deteriorate further over the time and worsen the pseudorange. Therefore, it becomes reasonable to consider clock-offset compensation as a time-varying case.

The main contribution of this paper is to propose compensation of the time-varying clock-offset in a LBL navigation. Specifically, the clock-offset dynamics are introduced to the ToFs as an autoregressive filter. Subsequently, interactions among the now biased ToFs and the kinematics of an autonomous underwater vehicle (AUV)-the navigation subject-are represented in a state-space form. Implementing the so-called graphic approach, minimum sensor requirement for this system's observability is then explicated. Finally, a standard discrete Kalman filter is deployed as the state estimator. In this paper, $\mathbf{I}$ and $\mathbf{0}$ denote identity matrix and zero vector, respectively. Their dimensions would vary according to the usage.

\section{PROBLEM STATEMENT}

Consider a LBL formed by $L$ acoustic transponders with known position, i.e. $\mathbf{r}_{1}, \ldots, r_{L} \in \mathbb{R}^{3}$, as shown in Figure 1. To mitigate the vertical dilution of precision (V-DOP) [18], one of the transponders is installed below a static vessel. Through a certain calibration procedure [19], the transponders' actual positions are in alignment with their nominal ones. An autonomous underwater vehicle (AUV) moves inside the baseline with a constant speed. At time $t$, each transponder sends an acoustic wave to the AUV receiver in a OWTT configuration. Once all the ToFs complete, the AUV used the pseudoranges to estimate its position and velocity at $t$, i.e. $\mathbf{r}(t) \in \mathbb{R}^{3}$ and $\mathbf{v}(t) \in \mathbb{R}^{3}$, respectively. Due to the factors mentioned earlier, clocks of the transponders suffer from clock-offsets and this in turn affects the localization accuracy.

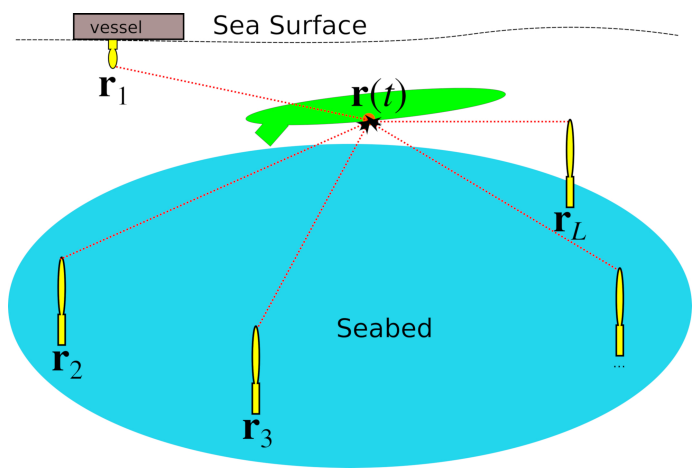

Figure 1. A LBL navigation involving $L$ transponders and an AUV

The above scenario holds on to the following assumptions. First, each signal carries encoded information about its send time and the transponder's identity [20]. Second, waves transmitted by transponders travel in LoS with constant speed. Third, all transponders' dynamics are identical. Fourth, the AUV clock is synchronous with the actual/absolute clock.

\subsection{Sampling period and time}

Since transponder has a certain update rate [21], it follows that a ToF measurement would take place every period of time. Therefore, it is of interest to describe dynamics involving the LBL and AUV in a discrete form. Accordingly, it may be defined that: 


$$
t:=k \tau
$$

where $\tau>0$ and $k \in \mathbb{N}$ denote sampling period and time index, respectively. Noticing that a ToF is initiated from transponder, it would then be sensible to take $\tau$ as the transponders' update rate.

\subsection{Vehicle kinematics}

The AUV position at $k$ can then be modeled as:

$$
\mathbf{r}(k+1)=\mathbf{r}(k)+\tau \mathbf{v}(k),
$$

while subsequently its velocity can be written as:

$$
\mathbf{v}(k+1)=\mathbf{v}(k),
$$

recalling that the AUV moves in a constant speed.

\subsection{Pseudorange and time-varying clock-offset}

A ToF measurement between transponder $j(j=1, \ldots, L)$ and the AUV at $k$ will result in:

$$
\left\|\mathbf{r}(k)-\mathbf{r}_{j}\right\|=c\left[t_{j}(k)-t_{0}(k)\right],
$$

where $c$ denotes the acoustic wave speed, while $t_{0}(k)$ and $t_{j}(k)$ are send and receive times of the wave transmitted by transponder $j$ at $k$, respectively. One should notice that $t_{0}(k)$ would be identical for all transponders following the third assumption. Introducing uncertainties into the ToF measurement while defining $d_{j}(k):=c\left[t_{j}(k)-t_{0}(k)\right]$, an expression for pseudorange based on (3) can now be written as:

$$
d_{j}(k)=\left\|\mathbf{r}(k)-\mathbf{r}_{j}\right\|+\theta(k)+\delta_{j}(k),
$$

where $\theta(k)$ denotes clock-offset in the transponder while $\delta_{j}(k)$ represents uncertainty due to the AUV movement during the ToF. For further elaboration about $\delta_{j}(k)$, the reader may consult [13] or the authors' previous work [14]. If the clock of transponder $j$ continues to drift from the reference clock, then $\theta(k)$ in (4) would also evolve over the time. For this time-varying case, $\theta(k)$ can be stated as:

$$
\theta(k+1)=\theta(k)+\tau_{c} \alpha(k)+w(k),
$$

where $\tau_{c}>0, \alpha(k)$, and $w(k)$ denote the clock-offset sampling period, clock-skew, i.e. instantaneous clockdrift rate [22, p. 7] and clock-offset's additional noise, respectively. Borrowing an approach developed in the wireless network community [23], a clock-skew is modeled as an autoregressive (AR) filter, i.e.:

$$
\alpha(m)=\sum_{m=1}^{P} a_{m} \alpha(m-1)+\eta(m),
$$

where $P$ and $a_{m}$ denote the AR's order and coefficient at $m^{\text {th }}$ order, respectively, while $\eta(m)$ represents an additional noise.

\subsection{States definition}

To obtain a state-space representation of the modeled system, it is defined that:

$$
\mathbf{r}(k):=\mathbf{x}_{1}(k), \quad \mathbf{v}(k):=\mathbf{x}_{1}(k), \quad \theta(k):=x_{3}(k), \quad \alpha(m):=x_{4}(k), \quad \cdots \quad \alpha(m-P):=x_{4+P}(k),
$$

noticing that the $P^{t h}$ order AR filter in (6) is now represented by $P$ state variables at $k$. Accordingly, (1)-(2) can be written respectively as:

$$
\mathbf{x}_{1}(k+1)=\mathbf{x}_{1}(k)+\tau \mathbf{x}_{2}(k)
$$

and:

$$
\mathbf{x}_{2}(k+1)=\mathbf{x}_{2}(k) \text {. }
$$


Furthermore, (5) can be written as:

$$
x_{3}(k+1)=x_{4}(k)+\tau_{c} x_{4}(k)+w(k),
$$

while (6) can now be stated as:

$$
\left\{\begin{array}{ll}
x_{4}(k+1) & =a_{1} x_{4}(k)+a_{2} x_{5}(k)+\ldots+a_{P} x_{x+P}(k)+\eta(k) \\
x_{5}(k+1) & =x_{4}(k) \\
& \vdots \\
x_{4+P}(k+1) & =x_{3+P}(k)
\end{array} .\right.
$$

\subsection{Pseudorange differences and additional states}

It is desirable to get and explicit relation between $d_{j}(k)$ and $\mathbf{x}_{1}(k)$ in (4). For this purpose, the so-called pseudorange difference approach [24] is to be implemented. The idea is to derive a difference between two pseudoranges, i.e. $d_{i}(k)$ and $d_{j}(k)$ based on (4), where $i=1, \ldots L$ but $i \neq j$. Much similar to the derivation steps in [15] and [25], carrying out mathematical manipulations on (4) leads to:

$$
\begin{aligned}
d_{i}(k+1)-d_{j}(k+1) & =\frac{d_{i}(k)+d_{j}(k)}{d_{i}(k+1)+d_{j}(k+1)}\left[d_{i}(k)-d_{j}(k)\right] \\
& -2 \tau \frac{\left(\mathbf{r}_{i}^{T}-\mathbf{r}_{j}^{T}\right)}{d_{i}(k+1)+d_{j}(k+1)} \mathbf{x}_{2}(k) \\
& +2 c \frac{\left[d_{i}(k+1)-d_{i}(k)\right]-\left[d_{j}(k+1)-d_{j}(k)\right]}{d_{i}(k+1)+d_{j}(k+1)} x_{3}(k) \\
& +2 c \tau_{c} \frac{\left(\left(d_{i}(k+1)-d_{j}(k+1)\right)\right.}{d_{i}(k+1)+d_{j}(k+1)} x_{4}(k)+\lambda_{i j}(k),
\end{aligned}
$$

where $\lambda_{i j}(k)$ represents all noises involved in the pseudorange difference derivation. To include all ToFs into the system's representation, it is defined based on (11) that:

$$
d_{1}(k)-d_{2}(k):=x_{5+P}(k), \quad d_{1}(k)-d_{3}(k):=x_{6+P}(k), \quad \cdots, \quad d_{L-1}(k)-d_{L}:=x_{4+P+U}(k),
$$

where $U=L(L-1) / 2$ is the number of possible combinations of pseudorange-differences derived from $L$ ToFs.

\subsection{State-space representation}

Incorporating (7)-(10) and (12), the state vector can now be defined as:

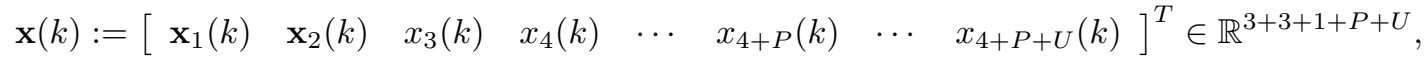

and subsequently the state-state space equations can now be written as:

$$
\left\{\begin{array}{l}
\mathbf{x}(k+1)=\mathbf{A}(k) \mathbf{x}(k)+\mathbf{w}(k) \\
\mathbf{y}(k+1)=\mathbf{C x}(k+1)
\end{array},\right.
$$

where

$$
\begin{gathered}
\mathbf{A}(k)=\left[\begin{array}{ccccc}
\mathbf{I} & \tau \mathbf{I} & 0 & \mathbf{0} & \mathbf{0} \\
\mathbf{0} & \mathbf{I} & 0 & \mathbf{0} & \mathbf{0} \\
\mathbf{0} & \mathbf{0} & 1 & \mathbf{A}_{34} & \mathbf{0} \\
\mathbf{0} & \mathbf{0} & 0 & \mathbf{A}_{44} & \mathbf{0} \\
\mathbf{0} & \mathbf{A}_{52}(k) & \mathbf{A}_{53}(k) & \mathbf{A}_{54}(k) & \mathbf{A}_{55}(k)
\end{array}\right] \in \mathbb{R}^{(3+3+1+P+U) \times(3+3+1+P+U)}, \\
\mathbf{A}_{34}=\left[\begin{array}{llll}
\tau_{c} & 0 & \cdots & 0
\end{array}\right] \in \mathbb{R}^{1 \times(P-1)},
\end{gathered}
$$




$$
\begin{aligned}
& \mathbf{A}_{44}=\left[\begin{array}{ccccc}
a_{1} & a_{2} & \cdots & a_{P-1} & a_{P} \\
1 & 0 & \cdots & 0 & 0 \\
\vdots & \vdots & \vdots & \vdots & \vdots \\
0 & 0 & \cdots & 1 & 0
\end{array}\right] \in \mathbb{R}^{P \times P}, \mathbf{A}_{52}(k)=-2 \tau\left[\begin{array}{c}
\frac{\mathbf{r}_{1}^{T}-\mathbf{r}_{2}^{T}}{d_{1}(k+1)+d_{2}(k+1)} \\
\vdots \\
\frac{\mathbf{r}_{L-1}^{T}-\mathbf{r}_{L}^{T}}{d_{L-1}(k+1)+d_{L}(k+1)}
\end{array}\right] \in \mathbb{R}^{U \times 3} \\
& \mathbf{A}_{53}(k)=c\left[\begin{array}{c}
\frac{\left[d_{1}(k+1)-d_{1}(k)\right]-\left[d_{2}(k+1)-d_{2}(k)\right]}{d_{1}(k+1)+d_{2}(k+1)} \\
\vdots \\
\frac{\left[d_{L-}(k+1)-d_{L-}(k)\right]-\left[d_{L}(k+1)-d_{L}(k)\right]}{d_{L-1}(k+1)+d_{L}(k+1)}
\end{array}\right] \in \mathbb{R}^{U} \\
& \mathbf{A}_{54}(k)=c \tau_{c}\left[\begin{array}{cccc}
\frac{\left[d_{1}(k+1)-d_{1}(k)\right]-\left[d_{2}(k+1)-d_{2}(k)\right]}{d_{1}(k+1)+d_{2}(k+1)} & 0 & \cdots & 0 \\
\vdots & \vdots & \vdots & \vdots \\
\frac{\left[d_{L-}(k+1)-d_{L-}(k)\right]-\left[d_{L}(k+1)-d_{L}(k)\right]}{d_{L-1}(k+1)+d_{L}(k+1)} & 0 & \cdots & 0
\end{array}\right] \in \mathbb{R}^{U \times(P-1)}, \\
& \mathbf{A}_{55}(k)=\operatorname{diag}\left(\frac{d_{1}(k)+d_{2}(k)}{d_{1}(k+1)+d_{2}(k+1)}, \quad \cdots \quad, \frac{d_{L-1}(k)+d_{L}(k)}{d_{L-1}(k+1)+d_{L}(k+1)}\right) \in \mathbb{R}^{U \times U}
\end{aligned}
$$

and

$$
\mathbf{w}(k)=\left[\begin{array}{lllllllllll}
\mathbf{0} & \mathbf{0} & w(k) & \eta(k) & 0 & \cdots & 0 & \lambda_{12}(k) & \cdots & \lambda_{(L-1) L}(k)
\end{array}\right]^{T} \in \mathbb{R}^{3+3+1+P+U} .
$$

On the other hand, the output part of (13), i.e. vector $\mathbf{y}(k)$ and matrix $\mathbf{C}$ are to be addressed separately in the next subsection.

\subsection{Observability analysis and minimum sensor requirement}

To become applicable for state estimation, it is a necessary that (13) observable. To examine the system's observability, the so-called graphic approach [26] is implemented. Here, an inference diagram is to be constructed by applying the following steps. The first step is to draw a node for each state variable in (13) while excluding the sampling index $k$. The second step-for each state equation- is to draw an arrow from state appearing at the left side directed to each state at the right. Results of these steps are shown in Figure 2.

The observability of a state variable is then checked using the following inspection routine on its representing node in Figure 2. First, if there is any incoming arrow towards the node, then it would be is assigned as a non-sensor node. Second, if there is no incoming arrow towards the node, then it would be assigned as a sensor node. In this sense, nodes $\mathbf{x}_{1}$ and $x_{5+P}, \ldots, x_{5+P+U}$ are assigned as sensor nodes. It means that deploying sensors is the only way to gather information about their respective states in (13). On the other hand, information about a state represented by a non-sensor node can be inferred from other state(s). In the sense of minimum requirement, this implies that measurement on a non-sensor state would not be a necessary for the system's observability.

Figure 2 seems to suggest that the observability of (13) requires $U+1$ sensor nodes. However, it should be recalled that $x_{5+P}(k), \ldots, x_{5+P+U}(k)$ states are formed by $L$ ToFs measurements to decipher relationship between ToFs and $\mathbf{x}_{1}(k)$ in (4). This means that once $L$ ToFs are available, $\mathbf{x}_{1}(k)$ can be observed through (12). Hence, the system actually only requires $L$ sensors to be observable and the output matrix can be written as:

$$
\mathbf{C}=\left[\begin{array}{lllll}
\mathbf{0} & \mathbf{0} & 0 & \mathbf{0} & \mathbf{I}
\end{array}\right] \in \mathbb{R}^{U \times(3+3+1+P+U)},
$$

i.e. the output vector would only consist the pseudorange difference states. 


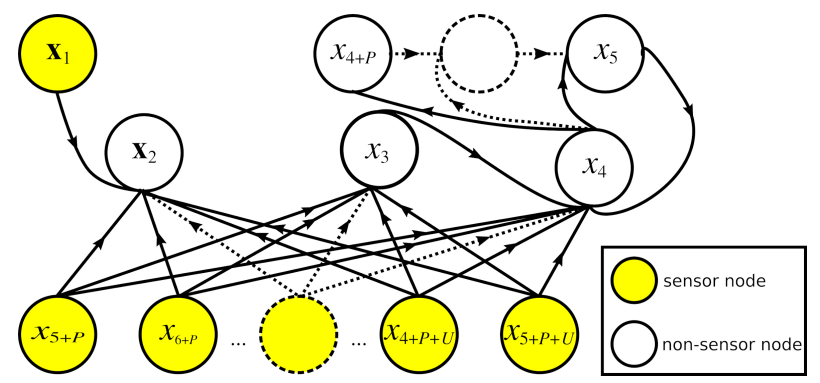

Figure 2. An inference diagram representation of (13)

\subsection{Proposed solution}

Considering that (13) is a linear system and all noises are modeled as Gaussian, a standard discrete Kalman filter [27] is to be implemented as the state estimator.

\section{SIMULATION AND RESULTS}

\subsection{Setup}

In this simulation, a LBL with five transponders is to be considered. The transponders' position are $\mathbf{r}_{1}=\left[\begin{array}{lll}0 & 0 & 0\end{array}\right]^{T} \mathrm{~m}, \mathbf{r}_{2}=\left[\begin{array}{lll}0 & 0 & 101\end{array}\right]^{T} \mathrm{~m}, \mathbf{r}_{3}=\left[\begin{array}{lll}1600 & 0 & 100\end{array}\right]^{T} \mathrm{~m}, \mathbf{r}_{4}=\left[\begin{array}{lll}0 & 1600 & 10\end{array}\right]^{T} \mathrm{~m}$, and $\mathbf{r}_{2}=\left[\begin{array}{lll}1600 & 1600 & 99\end{array}\right]^{T} \mathrm{~m}$. Here, it is assumed that $c=1500 \mathrm{~m} / \mathrm{s}$, while the LBL of choice is a low update rate type, i.e. $0.1 \mathrm{~Hz}$ [21]. In accordance to this rate, $\tau$ is set to be $10 \mathrm{~s}$.

Numerical values related to the clock dynamics are taken from [23], i.e. $\tau_{c}=900 \mathrm{~s}, P=5$, and:

$$
\left[\begin{array}{lll}
\hat{a}_{1} & \cdots & \hat{a}_{5}
\end{array}\right]=\left[\begin{array}{lllll}
0.9271 & 0.4613 & 0.07483 & -0.387 & -0.03118
\end{array}\right],
$$

where $\hat{a}_{1}, \cdots, \hat{a}_{5}$ are the estimated values of $a_{1}, \cdots, a_{5}$, respectively. Similar to [15], the initial value of the clock offset is set to be $0.3 \mathrm{~s}$ that would equal to $45 \mathrm{~m}$ deviation of a pseudorange.

The AUV is deployed to follow a helix trajectory with radius $250 \mathrm{~m}$ as shown in Figure 3 . AUV starts from $\mathbf{r}(1)=\left[\begin{array}{lll}949.47 & 707.852 & 5.085\end{array}\right]^{T} \mathrm{~m}$ with respective speed $\mathbf{v}(1)=\left[\begin{array}{lll}-0.0246 & 0.785 & 0.0085\end{array}\right]^{T}$ $\mathrm{m} / \mathrm{s}$ and is expected to finish at $\mathbf{r}(1000)=\left[\begin{array}{lll}950 & 700 & 65\end{array}\right]^{T} \mathrm{~m}$. The considerable large numbers of sampling is chosen to accommodate the evolution of $\theta(k)$ and $\alpha(k)$. At the estimator, initial values for the position and speed are set to be $\tilde{\mathbf{r}}(0)=\left[\begin{array}{lll}950 & 700 & 5\end{array}\right]^{T} \mathrm{~m}$ and $\tilde{\mathbf{v}}(0)=\left[\begin{array}{lll}0 & 0 & 0\end{array}\right]^{T} \mathrm{~m} / \mathrm{s}$, respectively.

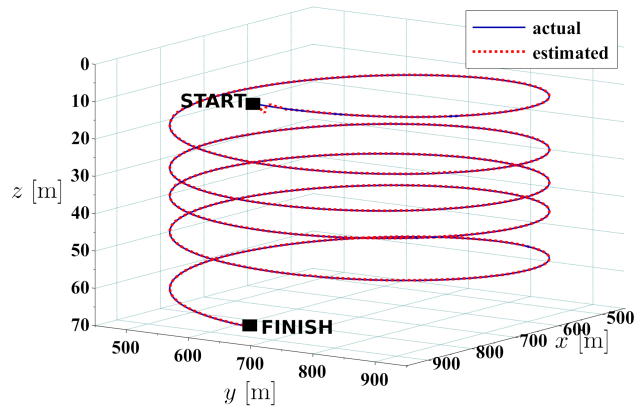

Figure 3. The AUV trajectory

\subsection{Results and discussion}

The simulation results are presented in terms of $t$ instead of $k$ where 1000 samplings would be equivalent to $10000 \mathrm{~s}$. In Figure 3, it is shown that the estimator can tracks the AUV actual position. A closer look on this particular result is presented in Figure 4 (a). In $x y z$ axis, it is shown that good accuracy are achieved during the trajectory tracking. On the other hand, a good result in velocity estimation is also the 
case as shown in Figure 4 (b). Regarding to the error profiles in Figure 4, it is worth to recall that the AUV velocities in $x$ and $y$ are not linear for a helix trajectory.

The above results indicate that the estimator manages to compensate the clock's biases. As shown in Figure 5 (a), the initially large clock-offset rapidly converges. Recalling the mathematical expression in (5), this convergence is also a result of the clock-skew's compensation. As shown in Figure 5 (b), the clock-skew also converges over the time in a rather similar manner to the clock-offset.

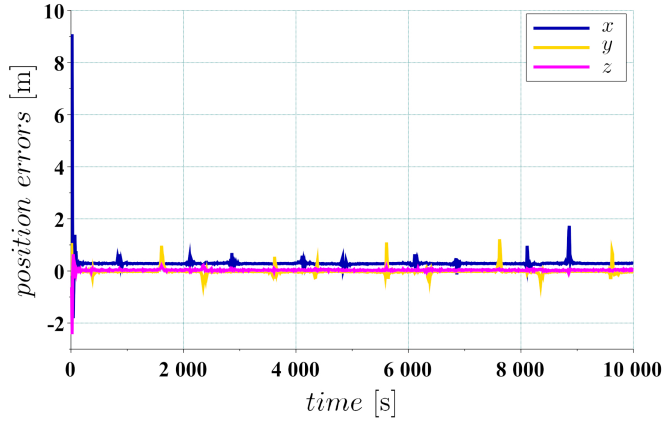

(a)

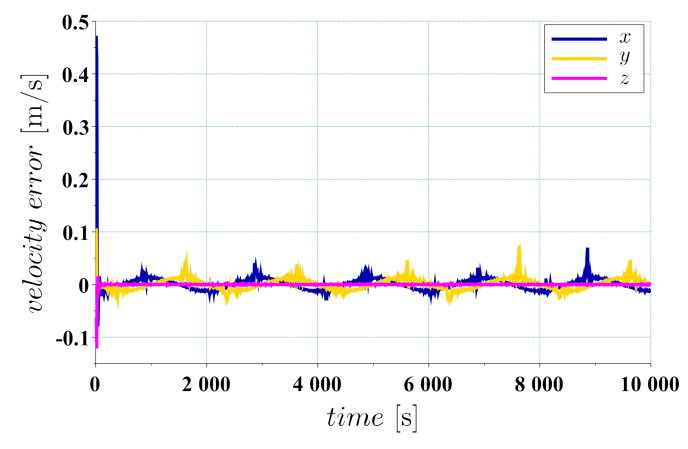

(b)

Figure 4. Estimation errors of the AUV kinematics in $x y z$ axes, (a) position errors, (b) velocity errors

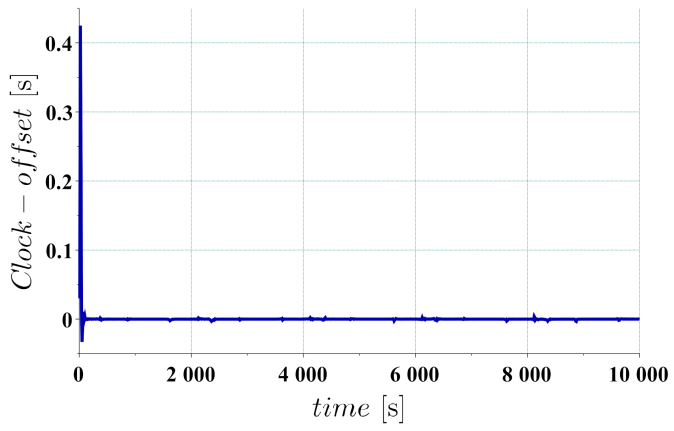

(a)

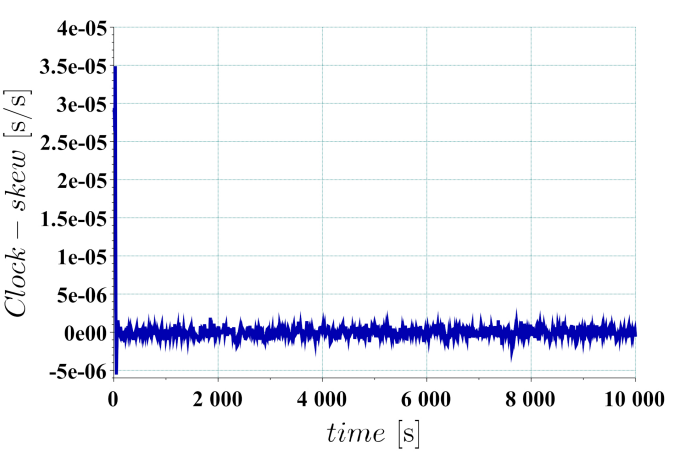

(b)

Figure 5. Compensation of the clock's biases, (a) clock offset, (b) clock skew

Furthermore, the estimation performances are presented in Table 1. Here, $\tilde{\mathbf{r}}=\left[\begin{array}{ccc}\tilde{r}_{x} & \tilde{r}_{y} & \tilde{r}_{z}\end{array}\right]^{T}$, $\tilde{\mathbf{v}}=\left[\begin{array}{ccc}\tilde{v}_{x} & \tilde{v}_{y} & \tilde{v}_{z}\end{array}\right]^{T}, \tilde{\theta}$, and $\tilde{\alpha}$ denote the estimated position, velocity, clock-offset, and clock-skew, respectively. Their respective averaged standard of deviation values are obtained through repeating the simulation procedure for more than 1000 times. It is shown that the estimator can provide position localization with accuracy less than $1 \mathrm{~m}$.

Table 1. Estimation performances.

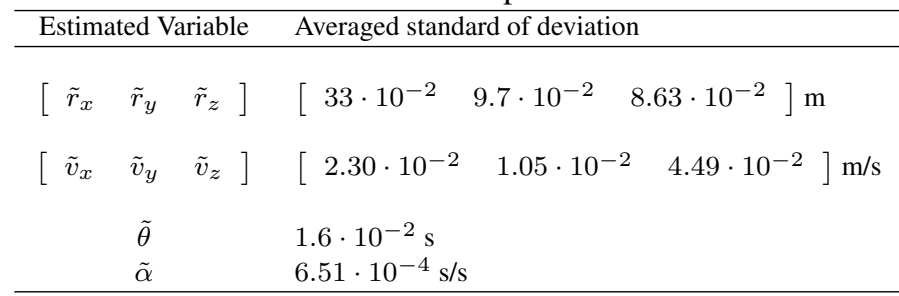




\section{CONCLUSION}

Compensation of clock-offset in a LBL navigation was presented in this paper. Its main contribution was to deal with the offset as a time-varying case by represent its dynamics as an AR filter. By simulation, it was demonstrated that the estimator manage to compensate the offset while provide localization to the AUV with less than $1 \mathrm{~m}$ accuracy.

\section{ACKNOWLEDGEMENT}

This work was sponsored by LPDP Indonesia under contract no. PRJ-5786/LPDP.3/2016.

\section{REFERENCES}

[1] L. Paull, S. Saeedi, M. Seto, and H. Li, "Auv navigation and localization: A review," IEEE J. Ocean. Eng., vol. 39, no. 1, pp. 131-149, 2014.

[2] I. Ahmad, "Geometric sensitivity of beacon placement using airborne mobile anchors," BEEI, vol. 8, no. 4, pp. 1333-1342, 2019.

[3] R. Almeida, J. Melo, and N. Cruz, "Characterization of measurement errors in a lbl positioning system," in OCEANS 2016, Shanghai, China, 2016, pp. 1-6.

[4] A. Bensky, Wireless Positioning Technologies and Applications. Artech House, 2007.

[5] M. Lévesque and D. Tipper, "A survey of clock synchronization over packet-switched networks," IEEE Comm. Surv. Tutor., vol. 18, no. 4, pp. 2926-2947, 2016.

[6] J. Breßler, P. Reisdorf, M. Obst, and G. Wanielik, "Gnss positioning in non-line-of-sight context-a survey," in 2016 IEEE 19th Int. Conf. Intel. Trans. Syst (ITSC), Rio de Janeiro, 2016, pp. 1147-1154.

[7] Y. Zhang, Y. Li, Y. Zhang, and T. Jiang, "Underwater anchor-auv localization geometries with an isogradient sound speed profile: A crlb-based optimality analysis," IEEE Trans. Wireless Comm., vol. 17, no. 12, pp. 8228-8238, 2018.

[8] Y. Li, W. Wang, M. Liu, S. Zhang, and J. Lan, "Underwater target tracking in three dimensional space based on sound speed profile," in 2019 CCC, Guangzhou, China, 2019, pp. 3502-3507.

[9] M. Ramdhan et al., "Measuring the underwater received power behavior for $433 \mathrm{mhz}$ radio frequency based on different distance and depth for the development of an underwater wireless sensor network," BEEI, vol. 8, no. 3, pp. 1059-1065, 2019.

[10] P. A. Miller, J. A. Farrell, Y. Zhao, and V. Djapic, "Autonomous underwater vehicle navigation.” IEEE J. Ocean. Eng., vol. 35, no. 3, pp. 663-678, 2010.

[11] Y. Chen, D. Zheng, P. A. Miller, and J. A. Farrell, "Underwater inertial navigation with long baseline transceivers: a near-real-time approach," IEEE Trans. Contr. Syst. Techn., vol. 24, no. 1, pp. 240-251, 2015.

[12] T. Gode, "Long baseline ranging acoustic positioning system," Master's thesis, Virginia Polytechnic Institute and State University, 2015.

[13] J. Yi, D. Mirza, R. Kastner, C. Schurgers, P. Roberts, and J. Jaffe, "Toa-ts: Time of arrival based joint time synchronization and tracking for mobile underwater systems," Ad Hoc Netw., vol. 34, pp. 211-223, 2015.

[14] Y. S. M. Simamora, H. A. Tjokronegoro, and E. Leksono, "Compensation of asynchronous time of flight measurements in a long baseline navigation," SN App. Sci., vol. 1, no. 3, p. 258, 2019.

[15] P. Batista, "Long baseline navigation with clock offset estimation and discrete-time measurements," Contr. Eng. Pract., vol. 35, pp. 43-53, 2015.

[16] J. R. Vig, "Introduction to quartz frequency standards," US Army Res. Lab., Tech. Rep. SLCET-TR-921B, 1992.

[17] F. L. Walls and J.-J. Gagnepain, "Environmental sensitivities of quartz oscillators," IEEE Trans. Ultrason. Ferroel. Freq. Contr., vol. 39, no. 2, pp. 241-249, 1992.

[18] R. Santerre, A. Geiger, and S. Banville, "Geometry of gps dilution of precision: revisited," GPS Solutions, vol. 21, no. 4, pp. 1747-1763, 2017.

[19] J. Zhang, Y. Han, C. Zheng, and D. Sun, "Underwater target localization using long baseline positioning system," App. Acoust., vol. 111, pp. 129-134, 2016.

[20] S. E. Webster, R. M. Eustice, H. Singh, and L. L. Whitcomb, "Advances in single-beacon one-way-travel- 
time acoustic navigation for underwater vehicles," Int. J. of Robot. Res., vol. 31, no. 8, pp. 935-950, 2012.

[21] J. C. Kinsey, Q. Yang, and J. C. Howland, "Nonlinear dynamic model-based state estimators for underwater navigation of remotely operated vehicles," IEEE Trans. Contr. Syst. Techn., vol. 22, no. 5, pp. 1845-1854, 2014.

[22] H. Y. Kim, "Modeling and tracking time-varying clock-drifts in wireless networks," Ph.D. dissertation, Georgia Institute of Technology, 2014.

[23] H. Kim, X. Ma, and B. R. Hamilton, "Tracking low-precision clocks with time-varying drifts using kalman filtering," IEEE/ACM Trans. Netw., vol. 20, no. 1, pp. 257-270, 2012.

[24] J. J. Caffery, "A new approach to the geometry of toa location," in Proc. IEEE 52nd VTC, vol. 4, Boston, MA, 2000, pp. 1943-1949.

[25] P. Batista, "Ges long baseline navigation with unknown sound velocity and discrete-time range measurements." IEEE Trans. Contr. Syst. Techn., vol. 23, no. 1, pp. 219-230, 2015.

[26] Y.-Y. Liu, J.-J. Slotine, and A.-L. Barabási, “Observability of complex systems.” PNAS, vol. 110, no. 7, pp. 2460-2465, 2013.

[27] G. Terajanu, Discrete Kalman Filter Tutorial. SUNY at Buffalo, 2013.

\section{BIOGRAPHIES OF AUTHORS}

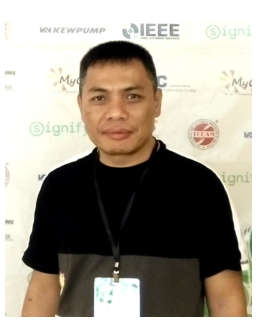

Yohannes S.M. Simamora obtained his Bachelor degree in engineering physics from Institut Teknologi Sepuluh Nopember (ITS) Surabaya and MS in instrumentation and control from Institut Teknologi Bandung (ITB) in 2002 and 2011, respectively. He currently is working toward the Doctorate degree in engineering physics at ITB. He has joined Politeknik Purbaya, Kabupaten Tegal (Indonesia) since 2005 where he is a lecturer at the Department of Mechanical Engineering. His current research interests include control engineering, nonlinear systems and control, mathematical modeling, sonar, and state estimation.

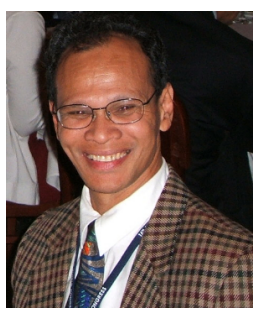

Harijono A. Tjokronegoro received his Bachelor degree in engineering physics from Institut Teknologi Bandung (ITB) in 1977; and subsequently DEA and PhD in adaptive systems, both from Institut National Polytechnique de Grenoble, France, in 1987 and 1990, respectively. He has been on the ITB faculty since 1977 and is currently Professor of Instrumentation and Control. His current research interests include linear sonar beamforming, circular dipping sonar, underwater acoustics beacon locator, and inertial navigation system and guidance.

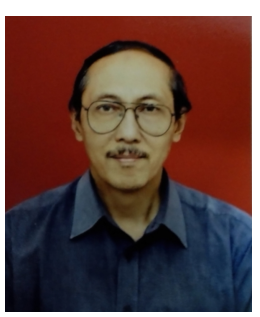

Edi Leksono obtained his Bachelor degree in engineering physics from Institut Teknologi Bandung (ITB), Bandung, M Eng. in control engineering from Tokyo Institute of Technology, Japan, and Dr.Eng. in electrical engineering from Keio University, Japan, in 1983, 1990, and 1998, respectively. He has been with ITB since 1983, where he is currently Associate Professor in Instrumentation and Energy System and the Head of Energy Management Laboratory at the Department of Engineering Physics. His research interests include robotics, instrumentation and control, energy systems, and battery management system for electric vehicles.

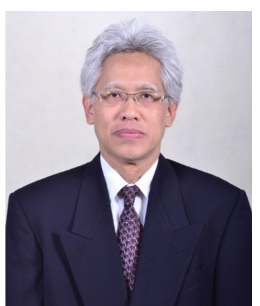

Irsan S. Brodjonegoro received his Bachelor degree in civil engineering from Institut Teknologi Bandung (ITB), MS in civil engineering from University of Minnesota, USA, and PhD in ocean engineering from University of Rhode Island, USA, in 1985, 1990, and 2001, respectively. He has joined ITB since 1987 as Assistant Professor at the Department of Ocean Engineering and currently serves as the Vice Dean for Academic Affairs at the Faculty of Civil and Environmental Engineering. $\mathrm{He}$ is also a lecturer at the Indonesia Naval College in Jakarta, Indonesia. His research interest is in the field of underwater acoustics, especially underwater acoustics propagation in deep ocean. 